\title{
¿Crisis o transformación del mundo laboral en las Américas?
}

Crise ou transformations du monde du travail dans les Amériques?

Transformations in the labor market in the Americas - or is it crisis?

\section{Donna Kesselman}

Traductor: Marta Gómez

\section{(2) OpenEdition}

\section{Journals}

Edición electrónica

URL: https://journals.openedition.org/ideas/3079

DOI: 10.4000/ideas.3079

ISSN: 1950-5701

Este artículo es una traducción de:

Crise ou transformations du monde du travail dans les Amériques ? - URL : https://

journals.openedition.org/ideas/1022 [fr]

Otras traducciones del artículo:

Transformations in the labor market in the Americas - or is it crisis? - URL : https:// journals.openedition.org/ideas/3073 [en]

Crise ou transformação do mundo dos negócios nas Américas? - URL : https://

journals.openedition.org/ideas/4722 [pt]

Editor

Institut des Amériques

Referencia electrónica

Donna Kesselman, «¿Crisis o transformación del mundo laboral en las Américas?», IdeAs [En línea], 5 | 2015, Publicado el 22 mayo 2015, consultado el 20 octubre 2022. URL: http:// journals.openedition.org/ideas/3079 ; DOl: https://doi.org/10.4000/ideas.3079

Este documento fue generado automáticamente el 20 octubre 2022.

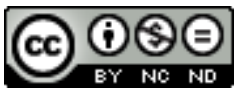

Creative Commons - Atribución-NoComercial-SinDerivadas 4.0 Internacional - CC BY-NC-ND 4.0 https://creativecommons.org/licenses/by-nc-nd/4.0/ 


\title{
¿Crisis o transformación del mundo laboral en las Américas?
}

\author{
Crise ou transformations du monde du travail dans les Amériques? \\ Transformations in the labor market in the Americas - or is it crisis?
}

\section{Donna Kesselman}

Tradución : Marta Gómez

1 ¿Hay una crisis que afecta al trabajo en las Américas? Si la hay, ¿en qué sentido es propia de este hemisferio en particular y cómo se manifiesta de Norte a Sur y en cada uno de los países? ¿O más bien cabría pensar que nos encontramos ante nuevas configuraciones que caracterizan a la globalización del mercado laboral del siglo XXI?

2 Esta es la pregunta que plantea este número de IdeAs, en línea con el número anterior dedicado a la «Crisis y efectos de la crisis en las Américas», tratando especialmente aquí los fenómenos relacionados con el mundo del trabajo.

3 La crisis del trabajo que afecta a los países industrializados desde hace una generación se ha convertido en una cuestión recurrente. La globalización financiera y las innovaciones técnicas y tecnológicas se combinan para eliminar las fronteras del comercio y la información, generando políticas públicas neoliberales que menoscaban las instituciones históricamente responsables de la regulación y la redistribución social. En lo que se refiere al mercado de trabajo y empleo, la Great Recession de 2008 no ha hecho más que acelerar el proceso en curso. Dentro de esta realidad de orden general, es necesario hacer algunas observaciones.

La globalización, lejos de ser homogénea, está hecha de estandarización y diferenciación (Azaïs C., 2010). El proceso de globalización del mercado laboral (globalizing world labor market) no es uniforme y dentro del mismo es necesario estudiar las particularidades nacionales y las de diversos niveles institucionales, así como la interacción entre agentes sociales múltiples (Kesselman D., 2010a; Giraud O., 2012). En lo que se refiere al trabajo, la diferenciación viene, por un lado, de las resistencias colectivas e individuales a las presiones de las empresas transnacionales para cada vez más flexibilidad y mayor reducción del coste laboral. Dichas resistencias se manifiestan 
en movimientos sociales en defensa del transporte público, la sanidad o la educación en un país tan dinámico como Brasil o, sorprendentemente, en la creación de derechos sociales en un país como Estados Unidos, donde algunos Estados federales imponen actualmente a las empresas -por primera vez en el caso de los poderes públicos- la atribución de vacaciones retribuidas a sus trabajadores (Thévenard E., 2015). Por otra parte, al ceder a las presiones de intereses locales, la flexibilidad favorece también la diferenciación.

Ciertamente, de Sur a Norte de las Américas existen realidades comparables, por no decir comunes: en el anterior número de IdeAs, Laurence Whitehead presentaba una serie de factores contextuales en defensa de una especificidad de experiencias en lo que puede llamarse el «Nuevo Mundo» (Whitehead L., 2013). Los países de América Latina comparten el hecho de pertenecer a los llamados «países del Sur»-The Global South-, un concepto que se inscribe dentro de la extensión del capitalismo. Esta denominación reciente respecto a la anterior de "países subdesarrollados» o de «Tercer Mundo» se refiere a las economías nacionales que no tienen acceso directo al mercado mundial. Anteriormente dominados e incluso colonizados por los países desarrollados, estos han soportado la prosperidad mercantil, y posteriormente capitalista, debido al traslado de sus propias riquezas para alimentar a las de los países del Norte. Actualmente, a algunos se les considera, según un lenguaje políticamente correcto, países en desarrollo, y a otros, países emergentes. A pesar de que el sentido de esta última categoría evoluciona rápidamente (Salama P., 2014), el sobre-entendido se presta a confusión.

6 Esta denominación, al igual que la de países en vías de desarrollo de la década de los 60 , implica la existencia de una trayectoria histórica lineal que seguiría el ejemplo de la de los países del Norte, que han tenido su propia revolución industrial y posindustrial. Sin embargo, esta idea de un esquema liso del crecimiento capitalista en las democracias occidentales tradicionales no obedece a la evolución de la división internacional del trabajo. Por otra parte, los entornos sociopolíticos propios de cada uno de los países del Sur y las tensiones que caracterizan al mercado mundial excluyen cualquier visión simplificada, por no decir, glorificada, de la historia económica.

7 La crisis de 2008 vendría a confirmar la heterogeneidad de las economías latinoamericanas. Como afirma Carlos Quenan, esta «realidad de trayectorias económicas diversas» se aplica incluso a países, en muchos aspectos, comparables, como México y Brasil. En este último, varios factores se conjugaron para atenuar el impacto de la crisis, mientras que en México, las consecuencias han sido especialmente «virulentas tanto para la economía como para la población». Los países que dependen principalmente de los ingresos de la exportación de hidrocarburos han reaccionado cada uno de forma distinta frente a la crisis (Quenan C., 2013).

8 Estas reacciones podrían explicarse por la existencia de modelos nacionales diferentes. Se cree que podrían existir incluso «variedades de capitalismo» (Hall P. y D. Soskice, 2001) específicas de América Latina, articuladas entre dos polos constituidos por dos países que se valen de ser grandes economías (Bizberg I., 2012; Théret B., 2002; Marques-Pereira J. y B. Théret, 2004) y se diferencian notablemente en el ámbito del trabajo. Según Bizberg, en Brasil se da un capitalismo de tipo State-led/inward oriented (guiado por el Estado con orientación interna), donde el Estado desempeña un papel central para orientar la actividad económica hacia el mercado interior, así como para fortalecer las bases de una integración activa en el mercado mundial. El artículo propuesto por Marcia Leite y Carlos Salas en este número de IdeAs parece confirmar 
esta hipótesis. Su estudio sobre la evolución de las profesiones da cuenta de una reducción de la desigualdad gracias al acceso creciente de poblaciones desfavorecidas a puestos de trabajo más cualificados, una tendencia que se impone a pesar de la crisis y que se inscribe dentro del modelo brasileño de desarrollo. En el polo y extremo opuesto está México, donde el Estado solo tiene un papel subsidiario para articular la estructura productiva, dirigida sobre todo hacia el exterior. Su acción se limita a regular y defender el capital nacional con la implementación de medidas contracíclicas en lo que puede llamarse un State regulated/externally-oriented capitalism (capitalismo regulado por el Estado con orientación externa). Esta percepción es también la de Élodie Segal, para quien la sociedad mexicana está «desfigurada por la desigualdad». Su análisis da cuenta de un modelo de producción que, en nombre de la modernización del país, está orientado según las necesidades de las finanzas y el clientelismo. Las presiones internas resultantes conducen a la desregulación, a la individualización y, para la población más frágil, al recorte de los derechos sociales de los trabajadores. Ciertamente, este es el caso de México, donde el salario mínimo está entre los más bajos de América Latina.

Otros países como Chile reúnen rasgos característicos de ambos modelos (Bizberg I., 2012). En estos términos se refieren a este país los autores Guillermo Wormald y Maria Paz Trebilcock. Su análisis de la relación entre trabajo y cohesión social en el medio urbano en Chile pone de manifiesto la emergencia de una «nueva sociedad mercantil» que vendría a subvertir las viejas relaciones y a imponerse cada vez más como un mecanismo de coordinación de oportunidades de acceso al trabajo. ¿En qué medida el trabajo sigue siendo un vector de creación de un espacio de encuentro, de valorización social y de ciudadanía? Mientras a finales del siglo pasado América Latina fue presentada como un posible «laboratorio social» del futuro del trabajo de los países del Norte -una postura retomada más tarde por Beck a través de su idea de la «brasileñización de Occidente» (Beck Ü., 2000)- en nuestra opinión, la experiencia chilena confirma nuestra hipótesis desarrollada en este número, y por otros autores, según la cual ciertas prácticas actuales en Estados Unidos relacionadas con la articulación del trabajo y las prestaciones sociales basadas en el mercado (market based) podrían invertir el sentido de la experimentación (Kesselman D., 2010b).

10 La afirmación de una globalización basada en la diferenciación -entre las economías y las sociedades y dentro de ellas- se justifica ampliamente respecto al mercado de trabajo y empleo. Las situaciones nacionales son difícilmente comparables en razón de las especificidades históricas de la construcción de cada Estado social y de un sistema de relaciones profesionales. A título de ejemplo, el carácter minimalista y fragmentado de las instituciones de reglamentación social en Estados Unidos da lugar a grandes diferencias entre regiones y empresas respecto al tipo de relaciones laborales (employment relationship). En conjunto, el caso estadounidense se aleja bastante del sistema de su vecino canadiense, mucho más protector (Lapointe P.-A., 2013; Kesselman D., 2007). En lo que respecta a los países del Sur, hay que tener en cuenta tanto la historia sociopolítica como las diferencias en el grado y estrategias de desarrollo.

11 Algunas contribuciones de este número se centran en las relaciones de trabajo y las luchas colectivas que, en su contexto nacional, buscan mitigar las consecuencias de la mundialización neoliberal. A pesar de su debilitamiento, el contrapoder del sindicalismo aún no ha dicho su última palabra.

12 Las fábricas de confección de ropa son uno de los principales sectores en los que se ha registrado un aumento del trabajo formal en Guatemala, sobre todo en el caso de las 
mujeres, en un país donde la gran mayoría de la población activa realiza actividades informales. Con todo, estas maquilas son ejemplo de precariedad y de las peores condiciones laborales, así como de feroces prácticas antisindicales. Pese a todo, las trabajadoras no se rinden ante los obstáculos y las barreras que diariamente se erigen contra el ejercicio de sus derechos sindicales, tal y como nos narra Quentin Delpech a través de investigaciones sobre el terreno.

Venezuela es un caso excepcional. Desde dentro del movimiento sindical, Thomas Posado nos ofrece una visión de los radicales cambios que han tenido lugar desde la instauración del régimen chavista. Este poder, que se apoya en los intereses populares y en una política de resistencia al dictamen del poder económico y financiero mundial, ha influido en el posicionamiento de las organizaciones obreras forjadas en el pasado y en la renovación del sindicalismo en el país.

IdeAs también presenta en este número un análisis comparativo del sindicalismo norteamericano. ¿Cómo se explica que Estados Unidos tenga una de las tasas de sindicalización más bajas del mundo occidental mientras en Canadá es de las más altas? En una entrevista, Paul-André Lapointe, especialista en relaciones laborales de la Universidad de Laval (Quebec), explica esta situación aparentemente paradójica dadas las similitudes socioeconómicas y la historia común que han acercado a los movimientos sindicales de ambos países: la instauración de la misma legislación de gestión de relaciones laborales en la industria y la constitución de las llamadas International Unions, esto es, las organizaciones sindicales implantadas tanto en Estados Unidos como en Canadá. El relato describe esta evolución cruzada y luego cada vez más divergente a partir de los años 60 , así como los retos a los que se enfrentan actualmente los trabajadores y sus organizaciones. El prisma de los derechos sindicales ofrece también un panorama de la variada cultura política y social que existe entre las distintas provincias canadienses.

Estas experiencias nacionales de crecimiento económico y defensa de los intereses sociales colectivos dan lugar a diferenciaciones en materia de igualdad social. Cualquier análisis de la situación en el y del trabajo, de Sur a Norte y dentro de cada sociedad particular, debe ir más allá de las estadísticas macroeconómicas. Las elevadas tasas de crecimiento y la extensión del trabajo asalariado en toda América Latina a pesar de la crisis cautivan a los vecinos del Norte. Estos hechos, sin embargo, no han traído consigo la construcción de una relación laboral asalariada que suponga prestaciones sociales del nivel que históricamente han alcanzado las de los países del Norte. También en esta región se ha registrado una multiplicación de empleos precarios (Giglia A., 2014; Rosenfield C., 2010).

16 Además, no por ello ha disminuido la informalidad en el subcontinente; como mucho, ha cambiado de rostro y de naturaleza (Azaïs C. y M. Pepin-Lehalleur, 2013). Guillermo Wormald y Maria Paz Trebilcock ilustran la complejidad del fenómeno en Chile: el empleo formal en forma de trabajo asalariado crece tanto en el caso de los hombres como en el de las mujeres, en todas las edades y en todos los deciles de ingresos, y ello, además, en forma de contratos indefinidos. Con todo, estos contratos, salvo en el último decil (10\% de los trabajadores asalariados mejor remunerados), distan mucho de ser igual de protectores que los que han tenido, por ejemplo, los trabajadores europeos.

17 En consecuencia, en el contexto de la mundialización neoliberal se plantea la cuestión de saber qué perspectivas sociales existen, incluso en economías abocadas a un 
desarrollo sin precedentes, y si el marco de redistribución social más extendido de los países del Norte está condenado a estrecharse inexorablemente.

Naturalmente, la historia aún no está escrita. Los artículos de este número de IdeAs contribuyen a replantear, en los términos más actuales, la pregunta del principio: ¿se puede hablar de una crisis del trabajo o nos encontramos más bien ante nuevas configuraciones, características de la globalización que augura el nuevo siglo (D’Amours M. et al., 2015)? La respuesta dependerá no sólo del juego de actores sino también de la salud del sistema.

Tras la crisis planetaria desatada por el hundimiento bursátil estadounidense de 2008, se anunció una nueva fase de recuperación. A comienzos de 2014, el diario francés Le Monde presumía de que «El FMI vislumbra[ba] una recuperación 'más fuerte y más prolongada'» (Guélaud C., 2014). Posteriormente, el FMI daba marcha atrás y, en vísperas del $45^{\circ}$ Foro Económico Mundial de Davos de enero de 2015, modificaba sus previsiones a la baja. El debilitamiento de la inversión y del crecimiento potencial en la mayoría de "grandes países» se afirma y la recuperación en la Zona Euro y Japón será más lenta de lo previsto. En cuanto a los países emergentes y en desarrollo, incluidos los BRICS, cuyo dinamismo fue tan alabado a principios de los años 2000, están atravesando un periodo difícil, sobre todo en el África subsahariana y América Latina. Estas dificultades se deben, entre otros factores, a la bajada de los precios de los productos básicos. Únicamente la economía de Estados Unidos ve sus proyecciones revisadas al alza (FMI, 2015).

Motor de la economía mundial, la economía estadounidense, que comienza a dinamizarse, debería aportar beneficios al mercado de trabajo, empezando por el del propio país. Con todo, la realidad es bien distinta, como escribe Donna Kesselman en su artículo. Según esta autora, el modelo de empleo estadounidense -el American Job Machine- está sumido en una crisis estructural. Olivier Frayssé plantea los retos en el largo plazo: la práctica desaparición de la jubilación por el sistema de reparto en Estados Unidos tiene consecuencias que van más allá del destino de esta prestación social, ya que revelan el eclipse de las bases mismas del sueño americano. Por último, Christian Azaïs propone una reflexión teórica sobre las transformaciones del trabajo en un mundo globalizado. ¿Se trata de una amplificación del proceso ya en marcha o bien de una crisis acompañada de un cambio de naturaleza?

21 Los lectores y lectoras de IdeAs deberán juzgar por sí mismos. Este panorama de rigurosas investigaciones planteado por dieciséis autores que trabajan en siete países de las Américas ofrece múltiples perspectivas de análisis sobre la evolución y la emergencia del mercado de trabajo y empleo, sobre el carácter cambiante de los fenómenos o sobre el papel de actores viejos y nuevos, indicadores todos del devenir de las sociedades y que alimentarán, esperamos, sus reflexiones. 


\section{BIBLIOGRAFÍA}

Azaïs, Christian, "Amérique du Sud : Travail ; Syndicalisme ", in Michel Bertrand, Antoine Coppolani, Isabelle Vagnoux, Jean-Michel Blanquer (dir.), Dictionnaire des Amériques, Paris, Laffont Collection Bouquins, publicación prevista en 2016.

Azaïs, Christian (dir), «Introduction», Labour and Employment in a Globalizing World: Autonomy, collectives and political dilemmas, Brussels, Peter Lang. 2010.

Azaïs, Christian et Pepin-Lehalleur Marielle (dir.), Modes de gouvernance dans quatre métropoles latino-américaines (Buenos Aires, Caracas, Mexico et São Paulo) : entre logiques institutionnelles et acteurs, Bruxelles, P.I.E. Peter Lang Ed., 2013.

Beck, Ülrich, The Brave New World of Work, Oxford, Polity Press, 2000.

Bizberg, Ilan, «Types of capitalism in Latin America», Revue Interventions économiques, $\mathrm{n}^{\circ}$ 47, 2012, p. 1-26, http://interventionseconomiques.revues.org/1772.

D’Amours, Martine, Soussi, Sid Ahmed, Tremblay, Diane-Gabrielle (dir.), Repenser le travail : Des concepts nouveaux pour des réalités transformées, Québec, Presses de l'Université de Québec, 2015.

Fondo Monetario Internacional (FMI), «Crecimiento mundial revisado a la baja, pese al abaratamiento del petróleo y la aceleración del crecimiento estadounidense», Boletín del FMI, 20 de enero de 2015, http://www.imf.org/external/Spanish/pubs/ft/survey/so/2015/NEW 012015AS.htm, consultado el 2 de abril de 2015.

Giglia, Angela, «Trabajo precario y redes de solidaridad. El caso de los gasolineros en la ciudad de México», in Angela Giglia y Adelina Miranda (coords), Precariedad urbana y lazos sociales. Una perspectiva comparativa entre México e Italia, México, UAM-Juan Pablos editores, 2014, p. 109-137.

Giraud, Olivier, « L'analyse scalaire des régimes d'action publique en Europe : l'apport méthodologique des comparaisons internationales ", Revue internationale de politique comparée, vol. $19, \mathrm{n}^{\circ} 2,2012$, p. 15-36.

Guélaud, Claire, «Le FMI entrevoit une reprise 'plus forte et plus large' », Le Monde, 8 avril 2014, http://www.lemonde.fr/economie/article/2014/04/08/le-fmi-entrevoit-une-reprise-plus-forteet-plus-large_4397717_3234.html\#ZAXV65cyXjha3dYj.99, consultado el 5 de marzo de 2015.

Hall, Per A., Soskice, David, Varieties of Capitalism, Oxford University Press, 2001.

Kesselman, Donna, « Travail et salariat aux États-Unis : Quels droits, quelles perspectives?», Revue Française d'Etudes Américaines, nº 111, 2007, p. 6-26.

Kesselman, Donna, «A Globalizing labour and employment market» (Postface), in Christian Azaïs (Dir.), Labour and Employment in a Globalizing World: Autonomy, collectives and political dilemmas, Brussels, Peter Lang, 2010a, p. 267-273.

Kesselman, Donna, «Trabalho precário e precarização institucional nos Estados-Unidos», Sociologias, vol. 12, $\mathrm{n}^{\circ} 25$, noviembre/diciembre, 2010b, p. 66-100.

Lapointe, Paul-André (Dir.), La qualité du travail et de l'emploi au Québec. Données empiriques et cadres conceptuels, Québec, Presses de l'Université Laval, 2013.

Marques-Pereira, Jaime et Théret, Bruno, «Mediaciones institucionales de regulación social y dinámicas macroeconómicas: los casos de Brasil y México» in Carlos Alba e Ilán Bizberg (Dir.), Democracia y Globalización en México y Brasil, México, El Colegio de México, 2004. 
Quenan, Carlos, «América latina frente a la crisis económica internacional: buena resistencia global y diversidad de situaciones nacionales», IdeAs, $\mathrm{n}^{\circ} 4$, otoño de 2013, http://

ideas.revues.org/780.

Rosenfield, Cinara (dir), «Trabalho, emprego e precarização social», Sociologias, vol $n^{\circ} 12, \mathrm{n}^{\circ} 25$, noviembre/diciembre de 2010.

Salama, Pierre, Des pays toujours émergents ?, Paris, La Documentation Française, 2014.

Théret, Bruno, Protection sociale et fédéralisme. L'Europe dans le miroir de l'Amérique du Nord, Montréal, Presse de l'Université de Montréal - Peter Lang, 2002.

Thevenard, Evelyn, «Paid Family Leave as Public Policy: A View from the States», in Gérard Gomez y Donna Kesselman (Dir.), Les femmes au travail dans les Amériques, Aix-en-Provence, Presses Universitaires de Provence (PUP), publicación prevista en 2015.

Whitehead, Laurence, «'Crisis' in the Americas: Is there a Regionally Distinctive Kind?», IdeAs, $\mathrm{n}^{\circ}$ 4, otoño de 2013, http://ideas.revues.org/611.

\section{AUTORES}

\section{DONNA KESSELMAN}

Donna Kesselman est Professeure des Universités à l'Université Paris-Est Créteil. Elle publie largement sur les questions du travail, de l'emploi et des relations professionnelles, en proposant une perspective comparative, (France, États-Unis, Brésil, Canada). Elle est co-rédactrice d'un recueil d'articles publié aux Presses Universitaires de Provence, articles issus des communications qui ont été faites lors du Congrès de l'IDA de 2013 "Les Femmes dans les Amériques", et plus particulièrement de l'axe thématique "Les femmes et le travail dans les Amériques". Elle a co-rédigé des études de la DARES sur les relations professionnelles. Elle est au bureau du RT 18 de l'Association Française de Sociologie traitant des relations professionnelles. 